\title{
Physical activity and its link to affective response: the choices might be crucial to psychological well-being
}

\author{
Atividade física e respostas afetivas: as escolhas parecem ser fundamentais para o \\ bem-estar psicológico
}

\section{AUTHOR'S \\ Thiago Sousa Matias ${ }^{1,2}$ (D) \\ Alexandro Andrade ${ }^{2}$ (D) \\ 1 Research Center of Physical Activity and Health at Federal University of Santa Catarina, Florianópolis, Santa Catarina, Brazil. \\ 2 Sport and Exercise laboratory at Santa Catarina State University, Florianópolis, Santa Catarina, Brazil.}

\section{CORRESPONDING}

Thiago Sousa Matias thiago.matias@ufsc.br

Campus Universitário Reitor João David

Ferreira Lima, Centro de Desportos,

Universidade Federal de Santa Catarina,

Trindade, Florianópolis, Santa Catarina.

Brazil. CEP: 88040-900.

\section{DOI}

10.12820/rbafs.23e0015

\section{(c) (i) (ㅇ)}

Copyright: This is an open-access article distributed under the terms of the Creative Commons Attribution License ${ }^{\circledR}$, which permits unrestricted use, distribution, and reproduction in any medium, provided that the original author and source are credited.
The view of physical activity (PA) as psychology health-promotion is relatively recent. Previous researches have tended to focus on neurophysiology a mechanism that tries to identify where positive outcome appears in the relationship between PA and mental health. In this sense, exercise has been categorized by its intensity and frequency ${ }^{1}$. We are call attention to the theoretical development of PA that has been constrained by over-relevance of biomedicine scientific paradigm, where studies have tended to address a direct and specific effect, excluding possible interpersonal, social and psychological variants that could influence related outcomes.

The present approach will intend to debate the relationship between PA and psychological well-being in a way that: 1) the subject is considered, preserving his intentions and choices, and 2) theoretical perspective provide a sound empirical basis for integrated account of emotional effects of PA. In Brazilian scientific context we observed that, in comparison to biological, and environmental factors, affective processes as determinants of PA behavior and the benefits of physical activity for psychological health have received considerably less attention ${ }^{1,2,3}$.

In 2014, Silva at $\mathrm{al}^{2}$ observed that there was a reduced body of knowledge regarding PA and mental health, also argued that the body of evidences were merely based on directional associations. Since then, the scenery seems to be the same.

It seems that we are stuck in the idea of replicating causal models, which has not helped in the understanding appropriately the matter or in the promotion of physical activity. We are call attention to the need of a new perspective on PA investigation. In the context of psychological well-being, PA should be understood as function of its affective consequences or anticipation of its affective response, a hedonic principle. In this cases, it is observed the importance of expected pleasure versus displeasure in determining PA adoption and maintenance, influencing relates to actual affective response ${ }^{3}$.

These principles are in line with the given trends of theories of motivation which is concerned to understand how individual behave in effective and healthy ways ${ }^{4,5}$. From a health perspective alone, investigations should be concerned about how to motivate to PA. This is a crucial matter, considering that PA can be an inherently rewarding activity that contributes to happiness and satisfaction. We observed from international literature that the extension of well-being is conducted as well as people feel deeper satisfied through psychological needs, consequently increasing motivation ${ }^{5,6}$. 
In this scenario we highlight an important consideration for exercise psychology context: PA can't be generalized. It is observed that the enjoyable feelings are what explain the reason why people provide engagement in sports activity as they find interesting, challenging and enjoyable. Contrarily to sports, exercises tend to be more extrinsically motivated. Choices on exercise settings might be supported by some gain or reward for being engaged on PA, such as health, to get fit or to perform other activities ${ }^{7}$. Exercises are more likely to have an instrumental focus concerning attractiveness and fitness and might be experienced as a strong social pressure, having a more external locus of causality. In this sense, this behavior might be accompanied by psychological ill-being ${ }^{8}$.

We are highlighting the need to look deeper into $\mathrm{PA}$ as its seems that the type of PA also matters. This might be crucial to avoid over-generalization in the relationship between PA and psychological well-being. Considering the extrinsic locus of motivation for some types of PA, it is observed in literature that a controlled environment that undermines satisfaction and pleasure is thought to decrease the provision of basic psychological needs such as autonomy, relatedness and competence, resulting in decrements of motivation and, subsequently, in maladaptive outcomes ${ }^{9}$.

We are not trying to determinate which activity will be beneficial or not, however, some modalities, such as sports, can naturally provide more internal experience, exposing people to happiness. When someone exercises in order to improve appearance or fitness, their motivation is extrinsic to the activity itself. Extrinsic is thus not a pejorative term, it merely refers to the goal of that activity being separable from the activity itself. The extension of exercises to be harmful seems to depend on the degree of internalization in the behavior ${ }^{7,8}$.

Further understanding is necessary to produce far-reaching and meaningful results. Indeed, more research is needed to better support PA in the context of mental health promotion. We would like to address some questions for future researches: for example, what is the affective source of different PA choices? Do different choices of PA predict a better psychological well-being? Do affective sources of PA mediate the relationship between PA and mental health?

The differences between choices of PA and its link to psychological status seems to be urgent to change par- adigms. The role of different PA choices, to the best of our knowledge, is rarely investigated. Considering different choices might permit us to better understand the importance of the affection on the relationship between PA and psychological outcomes. To be exposed to PA might not be easy, beholding many constrains on social, interpersonal and psychological setting, which appears to difficult the engagement and its maintenance overtime. Nevertheless, the general beliefs about the benefits of PA have led people to perform PA, often disregarding their intentions and pleasure of being engaged. In this context, it is signalized that the choices might matter.

\section{Conflict of interest}

The authors declare no conflict of interest.

\section{Authors' contributions}

Matias TS, conceived of the presented idea and wrote the text. Andrade A, conceived the overall direction and performed the review of the text.

\section{References}

1. Lopresti AL, Hood SD, Drummond PD. A review of lifestyle factors that contribute to important pathways associated with major depression: diet, sleep and exercise. J Affect Disord. 2013;148(1):12-27.

2. Silva J, Andrade M, Brito A, Hardman C, Oliveira E, Barros M. Atividade física e saúde mental: uma experiência na formação inicial em Educação Física. Rev Bras Ativ Fís Saúde. 2014;19(1):133-40.

3. Williams DM. Exercise, affect, and adherence: an integrated model and a case for self-paced exercise. J Sport Exerc Psychol. 2008;30(5):471-96.

4. DeciEL, Ryan,RM.Intrinsic motivation and self-determination in human behavior. New York: Plenum Press, 1985.

5. Deci EL, Ryan RM. The" what" and" why" of goal pursuits: Human needs and the self-determination of behavior. Psychol Inq. 2000;11(4):227-68.

6. Salmon P. Effects of physical exercise on anxiety, depression, and sensitivity to stress: a unifying theory. Clin Psychol Rev. 2001;21(1):33-61.

7. Ryan RM. Patrick H. Self-determination theory and physical activity: the dynamics of motivation in development and wellness. Hellenic J Psychol. 2009;6:107-24.

8. Frederick CM, Ryan RM. Differences in motivation for sport and exercise and their relations with participation and mental health. J Sport Behav. 1993;16(3):124.

9. Barkoukis V, Hagger MS, Lambropoulos G, Tsorbatzoudis H. Extending the trans contextual model in physical education and leisure-time contexts: Examining the role of basic psychological need satisfaction. Br J Educ Psychol. 2010;80(4):647-70. 